Mappemonde

Revue trimestrielle sur l'image géographique et les formes du territoire

$127 \mid 2019$

Varia

\title{
Nantes, les mues de la ville. Aperçu, quinze ans après
}

\section{Danielle Rapetti}

\section{OpenEdition}

\section{Journals}

Édition électronique

URL : http://journals.openedition.org/mappemonde/1924

DOI : 10.4000/mappemonde.1924

ISSN : 1769-7298

\section{Éditeur}

UMR ESPACE

\section{Référence électronique}

Danielle Rapetti, « Nantes, les mues de la ville. Aperçu, quinze ans après », Mappemonde [En ligne], 127 | 2019, mis en ligne le 03 mars 2020, consulté le 10 décembre 2020. URL : http:// journals.openedition.org/mappemonde/1924; DOI : https://doi.org/10.4000/mappemonde.1924

Ce document a été généré automatiquement le 10 décembre 2020.

\section{(c) (i) (2) (2)}

La revue Mappemonde est mise à disposition selon les termes de la Licence Creative Commons Attribution - Pas d'Utilisation Commerciale - Partage dans les Mêmes Conditions 4.0 International. 


\title{
Nantes, les mues de la ville. Aperçu, quinze ans après
}

\author{
Danielle Rapetti
}

Dans les années quatre-vingt-dix, la métropole estuarienne de renommée industrielle historique entre dans une phase de profondes mutations économiques et sociales et lutte pour tenir son rang dans la France de l'Ouest et progresser sur la scène européenne. Publié en 2004, l'article intitulé «Contre vents et marées: "Nantes Atlantique" » rend compte de cette évolution en deux temps : " Nouvelle donne » suivi de «La quête métropolitaine » (https://mappemonde-archive.mgm.fr/num2/articles/ art04205.pdf).

2 Nantes poursuit sa métamorphose à un rythme accéléré depuis le début du siècle, en profondeur et aussi de manière ostentatoire, à la façon d'une ville désormais extravertie. L'exposition "Rock une histoire nantaise » tient l'affiche depuis plus d'un an au musée du Château des ducs de Bretagne. Une "exposition unique pour comprendre comment la ville est passée du statut de belle endormie à celui de « Nantes l'effervescente » annonce le site Nantes-tourisme.com. Tout est dit. Ou presque. Dans un contexte de compétition permanente, nombre de villes partagent ces expressions flatteuses. Ainsi se réveillent d'autres belles endormies comme Angers, Bordeaux ou Reims. Une dynamique économique et culturelle incontestable renforce le pouvoir attractif d'une métropole qui, au demeurant, doit surmonter le handicap d'une situation marginale en Europe sur la façade atlantique ${ }^{1}$.

\section{Une « ville effervescente ». Air du temps et singularités}

3 Nantes cherche toujours à renforcer son rayonnement en conjuguant esprit d'innovation et richesse culturelle, patrimoniale ou futuriste. La Cité des congrès accueille la Folle journée, festival de musique classique de renommée internationale tout comme «Utopiales », festival de science-fiction, classé à ce titre au premier rang européen. 
4 Ses actions en faveur du développement durable et de la mise en valeur d'un cadre naturel très aéré, doté d'agréables vallées, d'un couvert végétal de 2900 hectares, dont 1050 ha d'espaces verts publics, valent à Nantes d'être élue Capitale verte de l'Europe en 2013. Le problème de la métropole est de concevoir des projets d'urbanisation aptes à accueillir 8000 habitants par an, dont $40 \%$, voire plus, dans la ville-centre, pour tenter de freiner l'étalement urbain tout en préservant son capital naturel. Nantes dispose encore au début du siècle de vastes friches, agricoles et industrielles, où naissent en dix à trente ans de nouveaux quartiers. Ces programmes ont en commun l'intention de favoriser la mixité sociale et la diversité des fonctions économiques et culturelles en des lieux bien desservis par les transports publics.

\section{La conquête de l'Est}

5 L'horizon 2020 verra s'achever, ou en voie de l'être, trois grands projets initiés entre 2000 et 2004. Dans le secteur est de Nantes arrivent environ 10000 habitants suite à la construction à terme de près de 5000 logements sur 80 hectares, répartis presque à égalité, entre l'opération Erdre-Porterie et la création de l'EcoQuartier BottièreChénaie en lieu et place d'anciennes tenues maraîchères. Une opération plus complexe - le Grand Projet de Ville Malakoff - Pré Gauchet - vise à restructurer un territoire de 164 ha, riverain du fleuve et de « La Petite Amazonie » classée Natura 2000, en associant " réhabilitation » de la zone urbaine sensible, construction de logements et de bureaux, équipements de tous niveaux et implantation de l'un des secteurs du centre d'affaires international Euronantes. Le quartier désenclavé se développe en lien avec l'île de Nantes au sud et la gare au nord, agrandie et modernisée pour répondre aux besoins d'un trafic de 25 millions de passagers en 2030 ; le double du trafic actuel. En point d'orgue de la renaissance du quartier, la nouvelle gare transfigurée par les architectes R. Ricciotti et C. Malleret, doit être livrée fin 2020.

6 Au sortir de la gare, le voyageur cherche le centre-ville, surpris parfois d'une large coupure - le cours de l'Erdre bitumé au pied de la tour de Bretagne - entre le plus ancien cœur historique, alentour de la cathédrale, du château, de l'église Sainte-Croix, et celui des XVIII ${ }^{\text {e-XIX }}$ siècles, riche quartier de la bourgeoisie d'affaires aux alentours de la place Graslin. Depuis près de vingt ans s'affiche la volonté d'implanter un «nouveau cœur de ville » enserré entre deux bras de Loire.

\section{L'« Île de Nantes », un chantier permanent}

7 «En 1987, lors de la fermeture des chantiers navals, lîle de Nantes était le symbole du déclin de la ville. Aujourd'hui, elle est devenue celui de son renouveau.». Ainsi s'exprime Jean-Marc Ayrault président de Nantes Métropole en 2009. Dix ans plus tard, réalisations et projets en cours répondent toujours à l'ambitieuse recherche de reconnaissance et d'attractivité européennes. Tenu à cette échelle pour l'un des chantiers majeurs du moment, ce territoire de 337 ha, où doivent vivre près de 40000 habitants en 2030, offre un paysage complexe comme chacune de ses phases d'urbanisation orchestrées par la Samoa, Société d'Aménagement de la Métropole Ouest-Atlantique, maître d'ouvrage, et confiées à trois équipes successives de maîtres d'œuvre (cf. illustration : île de Nantes, chronologie). D’amont en aval, la traversée de l'île conduit d'un lieu de prière - le couvent des Carmes - à un parc d'attractions où déambule un éléphant mécanique, soit: du secteur de Beaulieu en partie urbanisé à 
compter des années soixante-dix, à celui de la Prairie-au-Duc, anciennes friches portuaires, industrielles et ferroviaires, en passant par un jardin exotique dans les halles des anciennes Fonderies de l'Atlantique et du vieux faubourg « République » axé sur la première ligne de ponts.

Nantes au XXI siècle : le réel et l'imaginaire

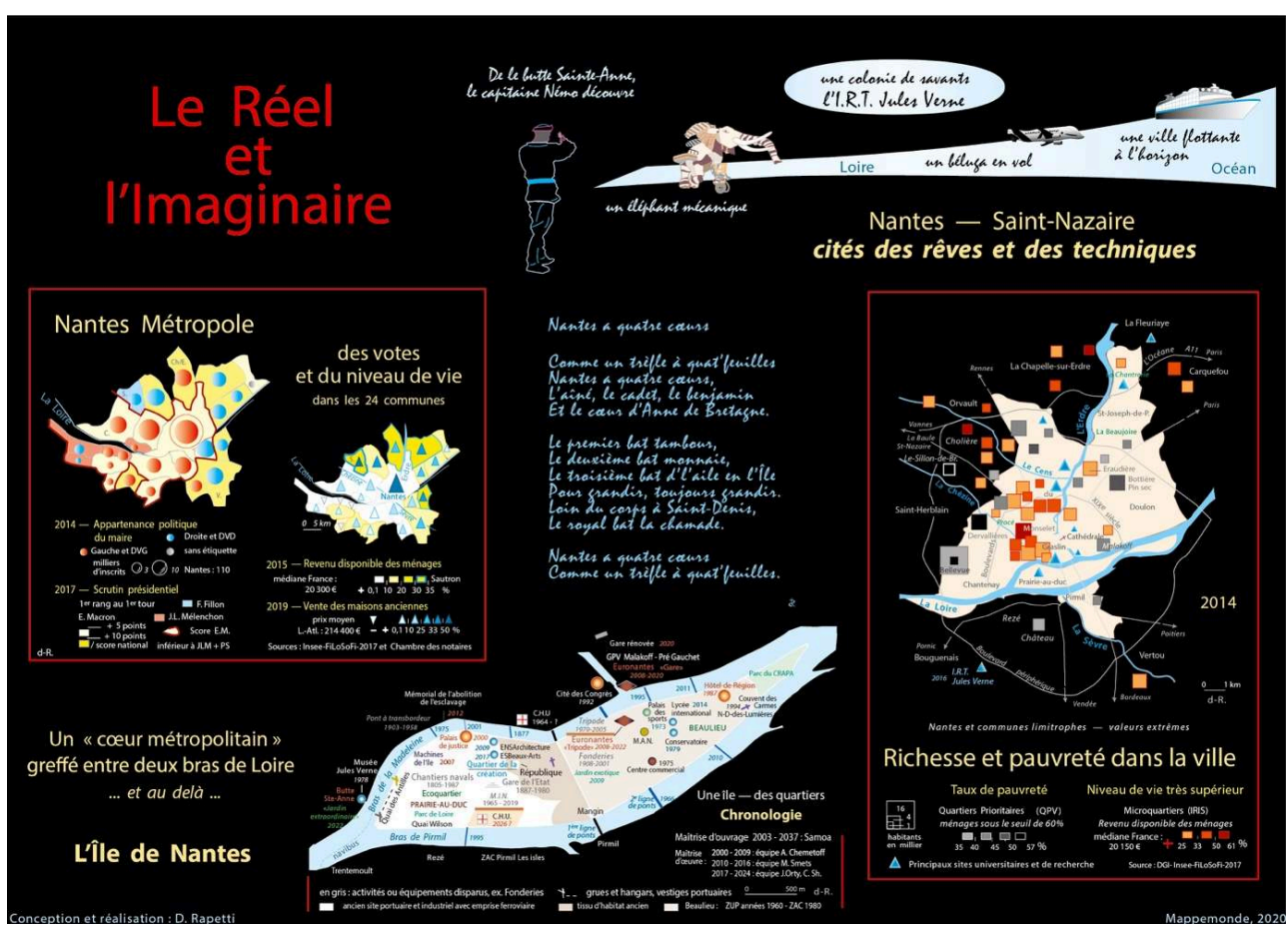

8 Au fil du temps s'opèrent sur l'île maints transferts d'activités. Le pouvoir politique régional s'y établit en 1987, le pouvoir judiciaire en 2000 dans un Palais signé Jean Nouvel, les médias Ouest-France et Télénantes en 2005 et 2017. Entre autres équipements, citons dans le domaine éducatif et sportif à Beaulieu, le lycée Nelson Mandela, à sections internationales, ouvert en 2014 à proximité du conservatoire de musique et du Palais des sports. À la pointe nord-ouest se joue le spectacle permanent de microquartiers en pleine mutation par effet combiné de constructions neuves et de réhabilitation d'éléments du patrimoine portuaire et industriel : hangars, halles Alstom et nefs des chantiers Dubigeon. Avec les Machines de l'île - Grand Éléphant et Carroussel des Mondes marins -, l'imagination a pris le pouvoir et le proclame en baptisant "Quartier de la Création » un petit territoire où la stratégie est de stimuler l'esprit d'innovation, les échanges entre universitaires, chercheurs, artistes et entreprises. Ainsi voisinent, par exemple, l'ENS d'Architecture, l'École des Beaux-Arts, le Pôle des Arts graphiques, l'École supérieure des métiers de l'audiovisuel, Médiacampus, la «Fabrique ", consacrée aux musiques actuelles, une pépinière de biotechnologies, un pôle universitaire dédié aux cultures numériques, etc. Le secteur sud de l'EcoQuartier de la Prairie-au-Duc, quant à lui, connaît un évènement majeur : le transfert à Rezé en 2019 du Marché d'intérêt national, prémisse d'une très lourde opération sur le terrain libéré : la construction d'un nouveau CHU devant regrouper en 2026 l'Hôtel-Dieu et l'Hôpital Laënnec. Ce quartier de la santé jouxtera immeubles et parc de Loire proches du quai Wilson et du quai des Antilles où, vestiges d'antan, deux grues surplombent les anneaux de Buren symboles d'ouverture à l'art contemporain. 


\section{Dessiner un « cœur métropolitain » toujours plus grand}

La conquête de l'île n'a pas pour seul objectif de contribuer à construire une nouvelle identité urbaine. La démarche s'inscrit dans une plus large perspective, celle du projet Rives de Loire, réaffirmé en 2005 : il faut multiplier les liens avec le fleuve. Ainsi se font face les deux sites du centre des affaires Euronantes, l'un proche de la gare et du stade Marcel Saupin requalifié, l'autre sur l'île dans le quartier du Tripode implosé où s'érige un ensemble architectural signé Christian de Porzamparc. Sur la rive sud, le projet pluricommunal Pirmil-les-Isles associera un pôle d'échanges majeur repensé - Pirmil - et l'unification d'un espace urbain morcelé. Au long du bras de la Madeleine, les liens sont historiques et imaginaires. La passerelle du Palais de Justice conduit au Mémorial de l'abolition de l'esclavage inauguré en 2012. Dans le Bas-Chantenay, l'un des hauts lieux de l'histoire ouvrière de la ville, un projet urbain répond toujours à la volonté d'accroître l'attractivité touristique, économique et résidentielle. C'est, par exemple, l'espoir de développer un pôle d'excellence dédié aux filières nautiques et navales. C'est aussi, au pied de la butte Sainte-Anne et du musée Jules Verne, la création d'un Jardin extraordinaire dans la carrière Miséry, ancienne friche des Brasseries de la Meuse, prêt à accueillir en 2022 un gigantesque Arbre aux Hérons, fruit de l'imagination de François Delarozière et Pierre Orefice, concepteurs des Machines de l'île de l'autre côté de l'eau. Tous ces projets liés à l'axe primordial de la Loire s'inscrivent dans le vaste périmètre d'« une nouvelle centralité » dessinée bien au-delà du secteur sauvegardé des deux cœurs historiques, qu'elle englobe, et de l'île de Nantes. Johanna Rolland, présidente de Nantes Métropole assume ce choix stratégique : «... on est en train d'imaginer le futur cœur de la métropole et on part pour un voyage de plus d'une dizaine d'années». (Cadre de ville, 2019).

\section{Les aléas de la croissance}

\section{Une stratégie urbaine controversée}

10 De tels bouleversements, spectaculaires sur les grandes zones réaménagées ou bien diffus sur l'ensemble de l'agglomération, engendrent des effets pervers ou bénéfiques.

\section{L'envers de la densification.}

11 Selon l'expression consacrée, la ville se renouvelle sur elle-même pour accueillir une population accrue. Un paysage urbain méconnaissable par prolifération d'immeubles dans un tissu pavillonnaire ancien n'est pas chose singulière, mais de grande ampleur à Nantes, avec comme exemple type la conquête en vingt-cinq ans des boulevards de ceinture du XIX ${ }^{e}$ siècle, de leurs maisons bourgeoises, ou modestes, entourées de jardins. Dans le secteur privé, « la densification urbaine (...) ne limite pas l'étalement urbain, parce qu'une grande partie des programmes sont achetés par des investisseurs plutôt que des accédants à la propriété » (Garat, 2009). Et les loyers sont élevés. Nantes "c'est l'étoile montante des classements des villes où investir ", parvenue au premier rang cette année, devant Toulouse et Rennes. (Net-investissement, 2019). Même réalité, autre point de vue : "Va falloir se calmer... spéculateurs et promoteurs ont la belle vie en ce moment à Nantes » lance un habitant de l'île lors d'une réunion de quartier (OF, 
2018). Les prix d'achat au $\mathrm{m}^{2}$ ont flambé : + $32 \%$ en moyenne en dix ans, plus qu'à Rennes et à Toulouse, moins qu'à Bordeaux et à Lyon.

\section{Des opérations trop coûteuses ou peu pertinentes ?}

créateur du Lieu Unique en 2000, souligne aux yeux du visiteur l'artère vitale entre les deux villes, jalonnée sur ses rives d'œuvres d'art pérennes ou éphémères. Grâce à une candidature coordonnée, à la volonté exprimée de «construire la ville autour du fleuve » et aux grands projets urbains associés - Ville Port à Saint-Nazaire, la Prairieau-Duc sur l'île de Nantes, "Estuaire " - la métropole bénéficie du label EcoCité en 2009 et, à ce titre, du Fonds d'Investissement d'Avenir (FIA) « Ville de demain », comme douze autres territoires sélectionnés suite à la mise en œuvre du Grenelle de l'environnement (actu-envir., 2009, 2011 et cohésion-t., 2013, 2017).

Tel un trait d'union symbolique, un vol de Beluga, l'avion-cargo d'Airbus, relie les usines de Bouguenais et de Gron, sauf en cas de transport terrestre ou fluvial. Les filières d'excellence, l'aéronautique et la navale, en phase actuelle ascendante, symbolisent le succès d'activités historiques converties aux nouvelles technologies. Les commandes d'Airbus, malgré l'échec commercial de l'A380, assurent l'emploi de 5700 salariés à Nantes et à Saint-Nazaire où l'effectif de Gron a triplé en dix ans.

Les « quartiers de la création » n'ont pas l'apanage de l'imagination, de l'innovation et d'échanges interdisciplinaires par ailleurs disséminés dans la ville au sein de centres de recherche, universitaires ou non. Deux exemples renommés en sont le pôle Biothérapie d'Atlanpole et l'IRT Jules Verne. Cet institut de recherche technologique mutualisé, le premier créé en France en 2012, s'implante à Bouguenais, à proximité du pôle de compétitivité EMC2 auquel adhèrent PME et grandes entreprises industrielles, telles que Airbus - et Boeing en 2017 -, DCNS, les chantiers navals, Béneteau et Renault. L'IRT supporte en particulier trois technocampus dédiés aux composites, à la robotique,

Mappemonde, 127 | 2019 
aux filières navales et énergies renouvelables (T. Océan), et à la digitalisation de l'industrie (Smart Factory à Saint-Nazaire).

Un soir d'hiver 2018, une « ville flottante » disparaît à l'horizon ; ce n'est pas le « Great Estearn » et Jules Verne à son bord, mais le dernier "Géant des mers » sorti des Chantiers de l'Atlantique. Selon le cycle habituel, la vie de l'entreprise oscille entre crise et prospérité. En 2010, les syndicats s'élèvent contre "une casse sans fin des emplois dans la navale » (EP, 2010); en 2019, il faut rouvrir l'école d'apprentissage. Les Chantiers, forts de 3000 salariés, peinent à embaucher. Le recours accru à la soustraitance et aux travailleurs détachés venus d'une dizaine de pays de l'est et du sud de l'Europe ne suffit pas pour honorer d'exceptionnels contrats : quatorze paquebots à construire d'ici 2026 pour les armateurs MSC, italo-suisse, et Royal Caribbean, américain ; sans compter d'autres navires, tels que quatre pétroliers ravitailleurs pour la Marine nationale, en collaboration avec Naval Group (ex DCSN). L'inquiétude vient d'ailleurs.

\section{Incertitudes et problèmes récurrents}

18 Les chantiers navals passent de main en main. Acker Yards en 2006 les acquiert d'Alstom, les cède en 2008 au Sud-Coréen STX, lequel les remet en vente en 2016. L'État soucieux de sécuriser l'emploi porte la part de capital acquise en 2008 de $33 \%$ à $50 \%$ lors de difficiles négociations avec le repreneur italien Ficantieri en 2017. Dans l'attente du rachat définitif soumis à l'autorisation des autorités de la concurrence européenne, l'État opère une nationalisation temporaire en 2018. La crainte porte aussi sur les liens entre Fincantieri et la Chine qui vient de construire son premier paquebot. Pour pallier les baisses de régime, les Chantiers se diversifient, multiplient les partenariats, misent sur la filière de l'éolien en mer, un marché plus porteur à l'étranger qu'en France où des procédures à l'encontre des parcs offshores bloquent les commandes. Toutefois, cet été 2019, le Conseil d'État valide le projet de parc éolien en mer de Saint-Nazaire - le premier en France -, puis celui de l'île d'Yeu-Noirmoutier. Les Chantiers, spécialistes de sous-stations électriques, construisent leur premier exemplaire destiné au marché français. Par ailleurs, le concept de navire de croisière "Silenseas " à voiles rigides «Solid Sail » ouvre d'autres horizons.

Après un sévère déclin, Le Grand Port maritime Nantes Saint-Nazaire quant à lui, renoue avec la croissance et retrouve presque en 2018 son record de 2005 (32,5 MT contre 34,5 T). L'activité reste toujours très sensible aux flux énergétiques. Par ailleurs, l'aide européenne permet d'ouvrir une nouvelle ligne d'autoroute de la mer entre Montoir et Vigo puis Tanger ; la liaison avec Gijon ayant échoué faute de rentabilité. Le port de Nantes dispose d'un hinterland trop restreint sans l'avancée de grands projets de desserte améliorée, notamment la transversale Rhône-Centre-Océan.

En peu de mots - « On condamne l'ouest de la France » $(E P, 2018)$-, le maire de SaintNazaire David Samzun exprime l'inquiétude d'une grande partie des milieux économiques et politiques. Le port, menacé en 2017 de relégation au niveau d'intérêt régional, conservera cependant le statut de port d'intérêt européen et international, le $4^{\text {ème }}$ par le trafic et le $1^{\text {er }}$ sur la façade atlantique. Mais le projet d'aéroport international est abandonné après des années de lutte des opposants au transfert à Notre-Dame-desLandes, écologistes et contestataires divers, en manifestations pacifiques ou violentes dans les rues de Nantes et sur la ZAD (Zone à défendre). Lors de la « consultation non 
contraignante» des électeurs de Loire-Atlantique en 2016, le «Oui » au transfert l'emporte à $55 \%$ (28\% des inscrits) sur le Non. À présent, l'aéroport de NantesAtlantique doit être réaménagé à Bouguenais, pour faire face à un trafic doublé en 8 ans (6 M de passagers en 2018).

21 De longue date, les villes du nord-ouest atlantique, dispersées sur un territoire excentré, tentent diverses formes de rapprochements afin de renforcer leur position sur la scène européenne, de faire reconnaître leurs compétences et de recevoir en retour les aides nécessaires à leur croissance. La démarche connaît maintes vicissitudes. Ainsi, dans le domaine universitaire, aucun campus en 2009 n'est qualifié « campus d'avenir ». En 2019, c'est l'échec, trois ans après sa création, de l'Université Bretagne Loire (UBL), communauté de 7 universités, grandes écoles et organismes de recherche de Bretagne et des Pays de la Loire. En revanche, en 2017, Nantes obtient, seule, le label d'excellence I-Site centré sur la santé et les industries du futur. Rennes pour sa part ambitionne de créer en 2019 une Grande Université unissant 7 grands établissements de la ville. Adoptée en 2015, la réforme territoriale maintient en l'état les deux régions Bretagne et Pays de la Loire. Les partisans de la Bretagne historique ne désarment pas. Suite à une pétition, le conseil départemental doit se prononcer en 2018: c'est Non au rattachement de la Loire-Atlantique à la Bretagne, mais Oui à l'organisation d'un référendum décisionnel par l'État. Cependant, les villes des deux régions non fusionnées en 2015 multiplient les coopérations au sein du Pôle métropolitain Loire-Bretagne créé en 2012 ; des coopérations qui n'excluent pas la compétitivité. L'arrivée du TGV en 1989 a joué un rôle prépondérant dans l'attraction de la ville. En 2017, le parcours total en LGV met Rennes à $1 \mathrm{~h} 25$ de Paris et Bordeaux à $2 \mathrm{~h}$, quasi à égalité avec Nantes. Face à cette rude concurrence, le territoire nantais doit renforcer ses atouts pour accueillir de nouveaux habitants.

\section{De l'aire urbaine à la métropole et à la ville-centre. Approche socio-démographique}

\section{Un territoire attractif à l'ouest de la France}

La bonne dynamique démographique persiste dans l'aire urbaine de Nantes. Un gain de population de $22 \%$ en 25 ans devrait permettre de franchir le seuil tant convoité du million d'habitants «à l'horizon 2030 ». Le rythme de croissance annuelle s'accélère entre 2011 et 2016, avec un taux de 1,5\% et un solde migratoire désormais supérieur au solde naturel (59\%). La métropole,où résident les deux tiers des 960000 habitants tient le $3^{\text {ème }}$ rang par son dynamisme $(+1,5 \% /$ an), derrière Montpellier $(+1,7 \%)$ et Bordeaux. Fait positif plus singulier, la ville-centre elle-même progresse $(+1,3 \%)$.

23 L'attraction résidentielle touche les étudiants, comme ailleurs, et diverses catégories d'actifs, mais Nantes est la seule métropole où les jeunes ménages avec enfants arrivent plus nombreux qu'ils n'en partent. L'attraction économique repose sur une offre d'emplois très diversifiée en forte augmentation $(+1 \% /$ an) comme à Toulouse ou Montpellier. Entre autres exemples parmi les activités innovantes, la filière numérique, bien développée aussi à Saint-Nazaire, emploie près de 25000 personnes dans une métropole labellisée French Tech, comme huit autres, dès 2014. Dans le domaine des biotechnologies, Eurofins Scientific connaît un essor spectaculaire. Crée en 1987 à Nantes, l'entreprise spécialisée dans les analyses d'aliments, de produits 
pharmaceutiques et de l'environnement, dirige aujourd'hui 150 laboratoires. Devenu leader mondial, le groupe conserve le site historique, mais... transfère son siège au Luxembourg en 2012.

Les composantes sociales évoluent avec le tissu économique et les coûts de l'immobilier. Les emplois de cadres des fonctions métropolitaines progressent à Nantes (15\%). Aux trois niveaux, la part des professions intermédiaires est identique ( $29 \%$ des actifs ayant un emploi), celle des cadres et professions intellectuelles supérieures passe de $20 \%$ dans l'aire urbaine à $27 \%$ dans la ville centre ; les deux cumulées surpassent désormais celles des ouvriers et employés (au mieux, respectivement, 19 et $26 \%$ dans l'aire urbaine). $\mathrm{Au} 3^{\text {zeme }}$ rang derrière les aires de Toulouse et de Lyon, Nantes affiche en 2015 un bon niveau de vie médian et le moins mauvais taux de pauvreté à $60 \%(10,2 \%)$, comparable à Rennes $(10,4 \%)$, nettement inférieur à la moyenne nationale $(14,2 \%)$. Ce taux monte dans la ville-centre $(16,2 \%)$ en lien avec le chômage $(17 \%)$. Toutes valeurs à nuancer à plus petits échelons d'observation.

\section{Des votes et du niveau de vie dans les communes de la métropole}

Les vingt-quatre composantes de la Métropole partagent un niveau de vie supérieur à la médiane nationale, une bonne participation au scrutin présidentiel du 23 avril 2017 et des votes Front National le plus souvent inférieurs à $10 \%$ des inscrits (5,7 \% à Nantes). Emmanuel Macron tient de loin le premier rang dans 19 communes sur 24 et, partout, son score dépasse la moyenne nationale de 2 à 13 points. La diversité communale apparaît comme un puzzle social toujours bien structuré, entre autres choses, par le réseau hydrographique et, de manière évolutive, par le réseau routier. En résumé, une diagonale nord-ouest/sud-est dessinée par les vallées de la Chézine et de la Sèvre sépare plusieurs types de territoires (cf. illustration : Nantes Métropole, des votes et du niveau de vie).

À l'Est, dans un cadre vert privilégié, revenu disponible et coût de l'immobilier très élevés vont de pair. En 2014, la droite conserve ou reconquiert les mairies. Emmanuel Macron trouve là ses meilleurs scores, 27 à $31 \%$ des inscrits. Deux cas se distinguent toujours : d'une part Sautron avec ses valeurs maximales de revenu, de prix de vente moyen des maisons anciennes et, en 2017, l'arrivée en tête de François Fillon (29,5\% des inscrits, à 1 point d'Emmanuel Macron); d'autre part La Chapelle-sur-Erdre où une population très aisée maintient le pouvoir municipal à gauche. La part des revenus du patrimoine y est moindre et celle des emplois métropolitains de haut niveau la plus grande $(22 \%)$, dont la moitié en fonctions de conception, recherche et prestations intellectuelles.

À l'ouest, de nouvelles populations recherchent la proximité des rives de Loire à des coûts immobiliers relativement moins élevés sur les anciens territoires ouvriers de l'estuaire industriel. Une certaine mixité sociale progresse. À l'échelon communal, le niveau de vie s'élève, assez peu toutefois au-dessus de la médiane. En 2017, la très grande majorité des inscrits place en tête Emmanuel Macron avec un score supérieur de 5 à 9 points à la moyenne. Cependant, à une exception près, trois éléments révèlent l'ancrage historique à gauche: la résistance, au prix d'alliances diverses, des municipalités en place dans la conjoncture défavorable de 2014, le premier rang tenu par Jean-Luc Mélenchon dans les anciens bastions ouvriers emblématiques et le vaste 
périmètre englobant les scores de La France Insoumise et du PS cumulés, supérieurs à celui d'En Marche.

Quant à Nantes où grandit l'audience des écologistes, le PS conserve la mairie et la présidence de Nantes Métropole en la personne de Johanna Rolland, grâce à une large alliance en 2014 (PS-EELV-PC-UDB). Mais, en 2017, près d'un inscrit sur quatre vote Emmanuel Macron, devant la gauche divisée (LFI : 20,3\% des inscrits, PS : 8,7\%). François Fillon, au $3^{\text {ème }}$ rang réalise un score supérieur à la moyenne (16,1 \%). L'échelon communal ne rend pas compte de la grande diversité sociopolitique des quartiers, en premier lieu du niveau de vie médian : $21300 € /$ an, soit un revenu disponible supérieur à la médiane nationale (20 $300 € /$ an), un peu moins élevé qu'à Toulouse et à Lyon, un peu plus qu'à Bordeaux et à Rennes.

\section{Richesse et pauvreté dans la ville}

29 Le 21 mai 2018, Presse-Océan publie deux articles comme en écho. L'un titre: "Immobilier haut de gamme, l'envolée »; l'autre: "SDF, les associations sont submergées ». Il y a à Nantes et dans les communes limitrophes, concentration des situations extrêmes au nord du fleuve, avec parfois proximité des contraires liée aux opportunités foncières. Le jeu des vallées, le cadre naturel, les voies de communication, l'histoire économique se conjuguent de longue date pour façonner une géographie des lieux de richesse et de pauvreté perceptible à l'échelon des microquartiers (cf. illustration : richesse et pauvreté dans la ville).

La banlieue nantaise s'est longtemps développée au sein même du très vaste territoire communal; en particulier les grands ensembles érigés dans les années 1950-1970 à la lisière du front pionnier d'urbanisation. Près de 54000 personnes vivent dans les quinze Quartiers Prioritaires de la Ville (QPV), à plus des trois quarts nantais. Le taux de pauvreté (à $60 \%$ ) oscille entre $35 \%$ au Château de Rezé à près de $49 \%$, voire plus de $50 \%$ aux Dervallières et au Sillon de Bretagne (57 \%) avec pour corollaire 40 à $50 \%$ des 15-64 ans au chômage. Des opérations de rénovation urbaine de longue haleine se succèdent; ainsi, pour les plus récentes, au Sillon-de-Bretagne à Saint-Herblain (2011-2014), aux Dervallières (2006-2024), à Malakoff (GPV 2001-2020). Quant aux quartiers nord, à Bottière-Pin Sec à l'est et à Bellevue, l'ANRU les intègre depuis 2014 à son nouveau programme au titre des 200 quartiers d'intérêt national en proie aux plus grandes difficultés. À Nantes aussi les voitures brûlent et les fusillades éclatent avec morts et blessés. "Ne laissons pas la violence tuer l'espoir dans les quartiers ", titre la banderole en tête d'une marche citoyenne organisée par les habitants de diverses cités ce 28 avril 2019 (Le Monde, 2019). La mairie annonce un investissement exceptionnel pour réaliser en dix ans le projet du «Grand Bellevue », ce quartier "sensible » le plus peuplé, construit il y a une cinquantaine d'années en marge de Nantes et de SaintHerblain.

De part et d'autre du parc de Procé, distants d'à peine deux kilomètres et dix minutes en bus, deux quartiers donnent une mesure a minima des inégalités sociales. Aux Dervallières, un ménage sur deux vit sous le seuil de pauvreté, au mieux un sur dix approche le niveau de vie médian en France, revenu disponible dépassé d'au moins $57 \%$ pour la moitié des ménages à Monselet, quartier emblématique de la grande bourgeoisie; les plus riches ici $(10 \%)$ disposent au minimum du revenu des plus pauvres là-bas multiplié par dix. Le centre ouest de la ville demeure toujours un secteur 
de forte concentration des richesses. À partir du cœur historique des XVIII ${ }^{\mathrm{e}}$ et $\mathrm{XIX}^{\mathrm{e}}$ siècles l'expansion des beaux quartiers a suivi l'axe nord-ouest de la route de La Baule et de la vallée du Cens, puis l'axe de la vallée de l'Erdre. À l'agrément du cadre de vie s'ajoute, depuis les années soixante, l'avantage de la proximité des sites universitaires et de recherche. La croissance de l'université et la création de nouveaux sites renforcent cet axe nord-sud appelé à présent «axe de la connaissance " tandis que, depuis quelques années, semble interférer au nord une relative attraction de l'autoroute A11, dite l'Océane.

Hors de ce schéma des extrêmes, traducteur des grands axes structurants de la morphologie sociale du Grand Nantes, bien des nuances distinguent les quartiers en incessante évolution. Une pauvreté diffuse subsiste hors des QPV. La flambée des prix de l'immobilier et certaines "réhabilitations" urbaines concourent à renforcer l'embourgeoisement du centre-ville et de ses abords, tel le vieux quartier ouvrier de Chantenay à présent très recherché. Les grands projets d'aménagement - le dernier en date vise à créer un ensemble de " hameaux » résidentiels sur le terrain de l'ancienne caserne Mellinet - restructurent le tissu urbain sur des dizaines d'hectares avant de générer de nouvelles relations de voisinage.

Comme un symbole des mues de la ville...

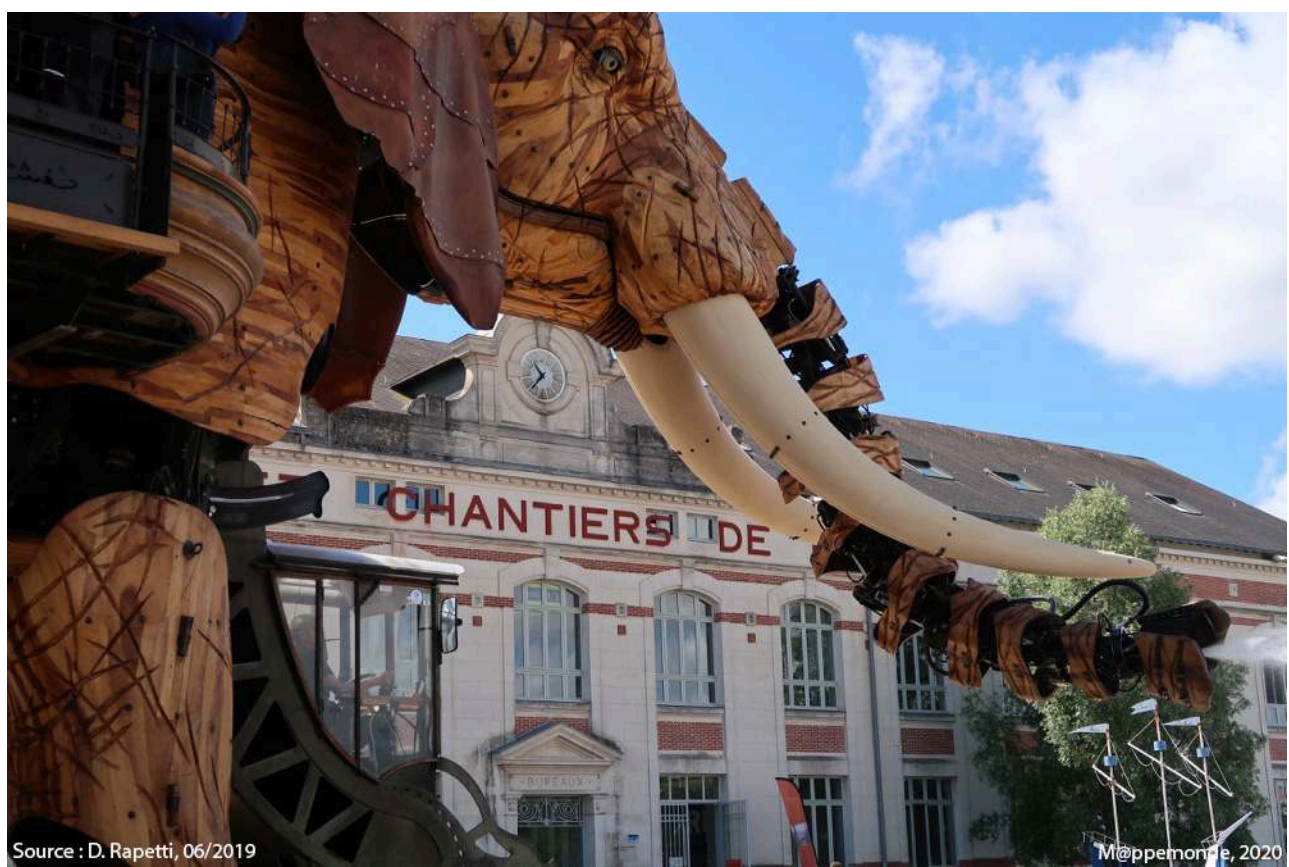

le Grand Éléphant des Machines de l'île, né en 2007, déambule devant l'ancien siège des Ateliers et Chantiers de Nantes, fermés en 1987. Le bâtiment accueille aujourd'hui la Maison des Hommes et des Techniques, le Centre d'Histoire du Travail et des activités de l'université de Nantes. En ce haut lieu de La Navale nantaise, rebaptisé Parc des Chantiers, se mêlent mémoire ouvrière, évènements culturels et attractions ludiques.

\section{L'épreuve du temps}

Loin de dresser un tableau exhaustif, cet aperçu atteste l'ampleur des mutations survenues ces quinze dernières années, notamment des opérations urbaines qui bouleversent le paysage nantais, à l'instar des quartiers neufs à l'est de la ville et sur 
l'île de Nantes, source d'enthousiasme comme de controverses. Soucieuse d'aménagements respectueux de la nature, Nantes entretient sa notable réputation de cité « verte et bleue » et reconquiert les rives de la Loire. Dans le même temps, la ville «se renouvelle sur elle-même ", se densifie sans parvenir à freiner ni la flambée des prix du foncier et l'étalement urbain, ni les inégalités sociales inhérentes au processus, quand bien même la métropole et l'aire urbaine ne comptent pas, en moyenne, parmi les plus défavorisées.

En phase de bonne dynamique économique, les grandes villes de l'Ouest confortent leur attractivité, jouent le partenariat afin de peser davantage à l'échelle européenne, mais le rude contexte de compétitivité conduit aussi à élaborer des projets indépendants ; à Rennes et à Nantes, par exemple, en ce qui concerne des institutions universitaires et de recherche. En revanche, les investissements d'avenir soutiennent en définitive des programmes de développement majeurs pour Nantes et Saint-Nazaire aux intérêts convergents. La dépendance eu égard aux choix stratégiques nationaux demeure toujours très grande et, pour partie, liée à la situation au sein de l'Europe. Les aléas du trafic portuaire sur la façade atlantique, les turbulences liées à l'abandon du transfert d'aéroport à Notre-Dame-des-Landes, suscitent l'inquiétude des milieux économiques soucieux de solide insertion dans les réseaux européens. Face aux incertitudes et à la concurrence, la métropole exploite ses atouts.

C'est une réalité, Nantes réussit sa reconversion industrielle, tient son rang de capitale régionale hyperactive en plein essor démographique. Dans le langage commun à toutes les métropoles, Nantes cultive les maîtres-mots - innovation, technologies, créativité, culture -, assortis de succès et porteurs de bonne renommée. Signe particulier: le pouvoir privilégié accordé à la mise en scène d'équipements et de manifestations ludiques et culturelles destinés à capter une clientèle touristique, si possible internationale, sans certitude de détenir en cela un solide facteur de métropolisation à long terme.

Hormis les mues spectaculaires de la ville, les plus profondes et la pertinence des stratégies qui les engendrent ne sauraient être appréciées dans leur totalité à trop court terme, voire même en 2030, date choisie pour l'exercice orchestré de prospective participative « Ma Ville demain » proposé aux habitants entre 2010 et 2012. Un quartier nouveau ou rénové ne trouve pas son équilibre, et son âme peut-être, avant longtemps ; a fortiori une opération urbaine composite de grande envergure comme l'île de Nantes ». Ce " cœur métropolitain » battra-t-il pour tous les habitants ? C'est-à-dire, entre autres choses, sera-t-il spontanément reconnu comme nouvel élément d'un centre-ville attractif pour des gens de toutes catégories sociales, de tous âges, dans un environnement harmonieux?

La prochaine décennie verra-t-elle réalisé le projet d'un nouveau franchissement, tunnel ou pont, véritable serpent de Loire proclamant l'urgente nécessité de pallier la saturation du pont de Cheviré ? Au-delà de l'agglomération nantaise, les deux rives de l'estuaire ne relèvent pas du même Schéma dit de Cohérence territoriale (SCOT), situation paradoxale peu propice à des aménagements communs, mais persistante lors de la création du Pôle métropolitain Nantes Saint-Nazaire, faute d'intégrer la communauté de communes du Sud-Estuaire. De rituelles manifestations ludiques et culturelles telles que la Biennale Estuaire ou la parade nautique Débord de Loire offrent une vision grand public d'union renforcée autour du fleuve. Simultanément entre les deux cités navales des alliances se nouent en profondeur, travaillent à renforcer un 
contexte économique dynamique, fort du savoir-faire technique des uns, de l'imagination et des recherches des autres. De là naissent des projets futuristes pour partie liés à la résurgence d'œuvres du passé. Tous les rêves ne deviennent pas réalité. Le projet très abouti d'un moderne pont à transbordeur n'a pas, à ce jour, été retenu par les pouvoirs publics. En revanche, Airseas, start-up liée à Airbus, doit industrialiser à Nantes un cerf-volant géant destiné à tracter les navires de commerce. Conçus par la start-up nantaise Néoline, les cargos rouliers à voiles rigides construits par le réseau de PME Néopolia assureront dès 2021 une liaison régulière entre Saint-Nazaire et la côte est des États-Unis, avec la clientèle de Renault, Manitou et Beneteau. Quant au projet Silenseas, la compagnie du Ponant, associée aux Chantiers de l'Atlantique, teste le prototype de voiles innovantes « Solid Sail » dès cette année sur son navire de croisière historique. Comme un rêve de voyages extraordinaires. Le monde imaginaire de Jules Verne au XXI ${ }^{e}$ siècle.

\section{BIBLIOGRAPHIE}

\section{Références citées dans le texte}

http://actu-environnement.com/, 4 novembre 2009 et 4 octobre 2011, Ecocités et écoquartiers, projets retenus.

http://cadredeville.com/, 10 janvier 2019, entretien avec Johanna Rolland.

https://www.cohesion-territoires.gouv.fr/, 2013 et 2017, Ecocités et villes de demain : 31 territoires soutenus par l'État.

EP : Écho de la Presqu'̂̂le, 30 avril 2010, « De nouveaux licenciements attendus... ».

EP : Écho de la Presqu'île, 17 janvier 2018. « Abandon de Notre-Dame-des-Landes ».

GARAT I. (2009), «L'emballement immobilier et ses effets urbains. L'exemple de Nantes ». Norois, $\mathrm{n}^{\circ}$ 212, p. 23-39

Le Monde, 28 avril 2019. « À Nantes, une marche inédite contre la violence qui tue l'espoir dans les cités ».

Le Moniteur, 5 janvier 2010. «Alexandre Chemetoff mal remercié à Nantes ».

Nantes Métropole (2013). « Ma ville demain, Nantes en 2030 ». Hors-série.

https://www.net-investissement.fr/ : « les 10 villes où investir en 2019 ».

OF : Ouest-France, 19 décembre 2009, « Remercié, l'architecte de l'île se rebiffe ».

OF : Ouest-France, 13 mars 2018, «Bataille du Futur CHU de Nantes : les points de frictions ».

https://sig.ville.gouv.fr/ : Atlas régional et départemental des Quartiers Prioritaires (QP). 


\section{Références documentaires sur la métropole. Sites en ligne}

https://auran.org/ : Agence d'Urbanisme de la région Nantaise, notamment « Les synthèses de l'AURAN".

http://www.fnau.org/fr/accueil/ : Fédération nationale des agences d'urbanisme (FNAU). Métroscope 2017 et Observ'agglo juin 2016.

https://www.insee.fr/fr/statistiques/1285709 : Analyse Pays de la Loire, nº 7, 2014. « L'aire urbaine de Nantes : un profil métropolitain singulier ».

http://www.nantes-amenagement.fr/ : Les projets de Nantes Métropole aménagement.

https://nantes.maville.com/ et https://www.nantesmetropole.fr/

https://www.nantes-citoyennete.com/ : Conseil de développement de Nantes métropole, contributions.

http://www.nantessaintnazaire.fr/ et http://www.polemetropolitainloirebretagne.fr/

http://www.poles-metropolitains.fr/ : réseau des pôles métropolitains.

http://www.resovilles.com/ et https://competitivite.gouv.fr/accueil-3.html

\section{Articles, rapports, ouvrages}

ENS (2012). Comptes-rendus de stage à Nantes. Département Géographie et Territoires. En ligne : http://www.geographie.ens.fr/Presentation,598.html

FRITSCH B. (2006). « Nantes-Saint-Nazaire, métropole exemplaire? ». L'information géographique, vol. $70, n^{\circ} 4$, p. 25-45.

GARAT I., dir. (2005). Nantes, de la belle endormie au nouvel Eden de l'Ouest. Paris : éditions Economica, $179 \mathrm{p}$.

MASBOUNGI A., dir. (2003). Nantes, la Loire dessine le projet. Paris : Éditions de La Villette, coll. « Projet Urbain », 193 p.

MASSON P. et al. (2013). Sociologie de Nantes. Paris : La Découverte, $126 \mathrm{p}$.

Place Publique - Nantes/Saint-Nazaire, la revue urbaine. Notamment « Les chroniques de l'île de Nantes ». En ligne : http://www.revue-placepublique.fr/

RAPETTI D. (2007). « Exode urbain des jeunes couples en Loire-Atlantique ». Étude périurbaine. Mappemonde, $\mathrm{n}^{\circ}$ 88, 2007-4, 17 p. En ligne : https://mappemonde-archive.mgm.fr/num16/ articles/art07405.html RENARD J. (2008). Nantes à la croisée des chemins. Rennes : PUR, $222 \mathrm{p}$.

RENARD J. (2016). « Le développement urbain de Nantes ». Fresques interactives - Ina avec reprise du thème de la quête métropolitaine. En ligne : https://fresques.ina.fr/auran/parcours/0001/ledeveloppement-urbain-de-nantes.html 


\section{NOTES}

1. Le titre fait référence à l'article paru dans le $n^{\circ} 74$ (2-2004) de Mappemonde: Rapetti D. (2004). «Contre vents et marées: "Nantes Atlantique ». $1^{\text {ère }}$ partie : « La nouvelle donne »; ${ }^{\text {ème }}$ partie : "La quête métropolitaine ».

2. Initiatrice dès 2010 d'une Lettre ouverte aux responsables locaux et nationaux, l'association Gaela - Groupement d'Analyses et d'Études de Loire-Atlantique - expose ses motifs d'inquiétude dans un document intitulé en 2016 « Le CHU sur l'île de Nantes : une catastrophe programmée?» Le collectif citoyen composé de médecins, professeurs émérites d'université et de spécialistes divers poursuit une « démarche d'alerte ». gaela-web.com

\section{INDEX}

Thèmes : 30 ans de publication dans Mappemonde

Mots-clés : France de l'Ouest, Nantes, Saint-Nazaire, métropolisation, urbanisme, étalement urbain, socio-géographie, île de Nantes 